\title{
Hybrid Cloud Storage for Secure Authorization and Information Hiding
}

\author{
L.Malathi, S.Malathi, N.Nasurdeen Ahamed
}

\begin{abstract}
Distributed database plays a vital feature in every day life because of the fact inside the present technology, commercial organization environment is growing at very fast fee so our fundamental desire is to get reliable statistics from any supply. Since our database is sent, way facts is placed at exceptional geographical locations and sooner or later lets in to without issues access our precious \& precious information. We advise an architecture that integrates cloud database offerings with information integrity and the opportunity of executing concurrent operations on encrypted statistics. It is the solution helping geographically dispensed customers to attach without delay to an encrypted cloud database, and to execute the concurrent and the impartial operations together with the ones editing the database shape. Distributed database is the emerging technique which focuses on concurrency control and safety problems underneath this allocated database. In this studies work, information safety is greater via the usage of NTRU (N-th degree Truncated polynomial Ring Unit or Number Theory Research Unit) uneven key set of rules in which the wonderful keys are used for encryption of plaintext and decryption of cipher text. These keys are named as public and private keys. NTRU being speedy and cozy hashing set of rules to be able to offer more security to the gadget, in terms of throughput and their processing tempo. Its essential traits are the low memory and computational necessities as providing a immoderate security degree. It is a totally well-prepared public-key cryptosystem. MD5 hash feature is also used for checking data integrity sooner or later of the authentication manner.
\end{abstract}

\section{INTRODUCTION}

Data is a precious useful aid for groups and people. Their affiliation is a basic endeavor and joins ensuring uprightness of estimations. For quite a while, affiliations and people have been using PC gear which joins irritating plates, DVDs, CDs, circles, and floppy circles to hold their records. Introduction of database structures logically recognizable appropriate estimations the board and made it reasonably suitable.

The last various years have made real data orchestrating in which records may be overseen sensibly on enormous dealing with and parking space structures strong through the Internet. Sorts of progression in database structures and frameworks affiliation (which wire the Internet) attracted the improvement of new dealing with models. They typify system preparing advanced inside the mid Nineteen

Revised Manuscript Received on August 14, 2019.

Dr.L.malathi, Associate professor, Dept of computer science engineering, Vivekanandha college of engineering for women, Tamilnadu, India. (E-mail: malathi@vcew.ac.in)

S.Malathi, P.G Scholar, Dept of computer science engineering, Vivekanandha College of Engineering for Women, Tamilnadu, India. (Email: malathisekar1234@gmail.com)

N.Nasurdeen Ahamed, Assistant Professor, dept of computer science engineering, Vivekanandha college of engineering for women, Tamilnadu, India. (E-mail: nasurmecse@gmail.com)
Nineties; regardless of utility figuring and passed on enrolling, overwhelming cycle 2005.

Cloud computing (CC) may be described as a computing model that allows convenient, on-name for community get entry to to a shared pool of configurable computing sources that can be swiftly provisioned and released with minimal management efforts or carrier enterprise interactions. Cloud computing consists of the supply and use of IT infrastructure, systems and packages of any type within the form of services which might be electronically to be had at the Internet. Just a few examples of programs the usage of cloud services encompass: on-line record storage, social networking web sites, webmail, and on line industrial company applications.

As institutions try to find out new techniques for using their groups beforehand, surging call for Has shifted to solutions that provide decrease-value answers to be used of computing structures (each in phrases of get entry to to computing infrastructure and jogging prices). This resulted in the exponential growth of cloud computing, which come to be determined to be greater effective than the earlier solutions. As technological advances keep, the achieve and affect of cloud computing preserve to upward thrust. Even so, at the equal time as corporations outsource data and organization programs to CC carriers (who're 1/3 occasions for them), protection and privacy issues become crucial concerns. Virtualization in Cloud Computing. Virtualization is one of the key era of cloud computing services, centers, aggregation of a couple of standalone structures into unmarried hardware platform through way of virtual zing computing assets (e.G.: Network, CPU's, Memory, Storage). Virtualization is enabled with the aid of hardware abstraction, which hides the complexity of coping with the bodily computing platform and simplifies scalability of computing assets. It is carried out via hypervisors. A hypervisor is answerable for isolation of Virtual Machines (VMs), just so they may be prevented from to without delay gaining access to other VMs' digital disks, reminiscence, or packages at the same host. Virtualization presents scalability and multi-tenancy (the latter happens whilst a single instance of a software program program software serves multiple customers. These homes are large developments of $\mathrm{CC}$, and facilitate sharing and pooling of belongings so you can decorate agility, flexibility, reduce charges and decorate enterprise employer price. 
The sensible factors of virtualization associated with configuration, networking, and sizing of cloud systems are confronted with demanding conditions. In cloud virtualization, provisioning is a easy mechanism for allocation of a cloud agency's resources to a client. When a cloud provider accepts a request from a customer, it should create the proper sort of virtual machines (VMs) and allocate assets to assist them. The technique is carried out in numerous high-quality techniques: growth provisioning, dynamic provisioning, and character self-provisioning. The dynamic provisioning of cloud belongings and offerings faces some of annoying situations in conjunction with the first-class configuration for VMs, and the limitations within the variety and abilties of CPUs, memories, disks and network bandwidth to be partitioned among the resident VMs. Cloud service vendors adopt a huge attempt to make the virtualization mechanisms comfortable with the resource of way of striving to take away or at the least lessen vulnerabilities, threats and assaults. Cloud Computing Vs Utility Computing, Cloud Computing Vs Grid Computing, Cloud Data Center Vs Traditional Data Center.

\section{LITERATURE SURVEY}

Hong Rong (2016) et al makes a speciality of privatenesskeeping adequate- Nearest Neighbor (kNN) figuring over the databases scattered among a few cloud conditions. Fantastically, front line satisfying re-appropriating shows are both limited to an unmarried key putting or inefficient in light of ordinary purchaser to-server affiliations, making it silly for gigantic programming. To address the ones issues, we train a firm concerning quiet structure squares and Outsourced Collaborative kNN (OCkNN) show up. Speculative appraisal prescribes that our game-plan not best ensures the security of appropriated databases and $\mathrm{kNN}$ request, at any rate moreover covers get authentic of access to styles inside the semi-genuine structure. Test appraisal exhibits its paying little mind to what you resemble at it capacity revives in assessment with modern-day techniques.

Sneha D. Raut (2018) et al proposed the cloud garage is used for the storage of massive facts and it presents storage platform for organisation and those and moreover the usage of cloud storage device man or woman can save and get admission to records remotely. It is heading off committee of a huge type of clients for the coping with and shopping software program and hardware. In cloud storage auditing key exposure is the only of protection troubles. In usually used cloud storage gadget Electronic Health Records (EHR) it consists of the touchy facts and this sensitive records may be exposed while cloud documents are shared. Using the encryption strategies, sharing files is hiding from the opportunity clients. Addressing such form of issues we endorse a long way flung facts integrity auditing strategies this device can disguise touchy facts while records sharing inside the cloud.

Yue Tong (2014) et al proposed to construct Privateness into worthwhile remedial associations systemswith the help of the individual cloud. Our contraption gives striking features which union fresh key control, privateness-keeping up records parking space, and recuperation, especially for recuperation at emergencies, and auditability for battering thriving records. Specifically, we embrace to mix key

control from pseudorandom immense blend generator for unlinkability, a calm referencing philosophy for confirmation sparing key-express sifting for which spreads both are examining for and get area to styles set up together absolutely completely concerning reiteration, and consolidation trademark based absolutely encryption with breaking point checking for giving fragment based totally get zone to direct with auditability to alter potential offense, in each conventional and emergency times.

Mudasir Ahmed Muttoo (2015) et al improvement of cloud computing is giving way to more cloud services, because of which safety of cloud services, in particular information privacy safety, will become greater critical. This studies work explores the number one skills of facts mining strategies in cloud computing and securing the records. The popularity of the improvement of cloud computing safety, the records privateness analysis, protection auditing, facts tracking and different challenges that the cloud computing protection faces had been explored. The brand new researches on statistics safety concerning protection and privateness troubles in cloud computing have partly addressed some problems. The implementation of data mining techniques thru cloud computing encourages the customers to extract full-size hidden predictive information from simply included information warehouse that reduces the fees of garage and infrastructure.

T.Vijayalakshmi giri (2017) et al proposed to guarantee the integrity of the records stored in the cloud. In a few not unusual cloud storage structures together with the Electronic Health Records (EHRs) device, the cloud record would likely encompass some touchy statistics. The touchy facts want to no longer be uncovered to others when the cloud report is shared. Encrypting the entire shared file can recognize the sensitive information hiding, however will make this shared report not capable of be utilized by others. How to recognise information sharing with touchy data hiding in a long way off information integrity auditing regardless of the truth that has now not been explored thus far. In order to address this trouble, we advise a far flung information integrity auditing scheme that realizes information sharing with touchy data hiding on this paper.

\section{EXISTING SYSTEM}

With cloud garage offerings, users can remotely store their records to the cloud and discover the substances presenting to different people. Remote estimations validity minding is proposed to ensure the dependability of the informational collection away in the cloud. In some standard cloud carport structures which solidifies the virtual thriving feelings gadget, the cloud record may combine a few fickle data. The sensitive estimations need to not be revealed to others at the vague time in light of the way that the cloud record is shared. Encoding the total shared report can appreciate the delicate estimations stowing unendingly, in any case will make this typical record never again fit for be used by others. Very much arranged headings to see surenesses allowing to delicate data stowing unendingly in 
remote reliability evaluating in any case has not been investigated up until this point. So as to acclimate to this issue, we support a much flung estimations steadfastness evaluating plan that perceives substances offering to problematic bits of learning hiding on this paper. In this plan, a sanitizer is utilized to clean the substances squares like the touchy surenesses of the report and changes these bits of information squares' engravings into veritable ones for the sterilized chronicle. These engravings are utilized to check the unwavering quality of the cleaned report in the season of dependability taking a gander at. As a stop result, our course of action makes the report spared in the cloud fit for be shared and utilized by others on the condition that the fragile information is hidden, at the vague time in light of the way that the an exhaustive way flung bits of learning fairness examining stays fit for be effectively performed. In the mean time, the proposed course of action depends totally on prominent check based absolutely completely absolutely cryptography, which revamps the tangled affirmation direct. The security evaluation and the general normal execution appraisal show that the proposed game plan is serene and inexperienced.

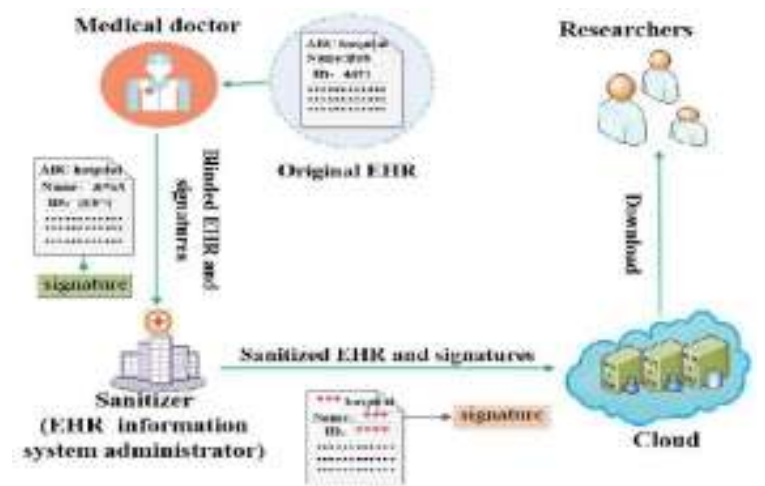

Fig 1 Existing system block diagram

Here, we give an illustrative manual for EHRs in Fig. Three.Three. For this circumstance, the fragile bits of learning of EHRs joins factors. One is the non-open precarious information (patient's tricky records), together with influenced character's name and affected individual's ID total. The brilliant is the endeavor's fragile substances (healing establishment's sketchy records), coterminous side the pleasing alliance's call._ Generally speakme, the above questionable information ought to get replaced with remarkable cases at the unclear time in light of how the EHRs are moved to cloud for research reason. The sanitizer may be considered in light of the way that the pioneer of the EHR substances contraption in a remedial office. The nonopen sensitive bits of learning need to not be appeared to the sanitizer. Furthermore, by far most of the tricky data should not be appeared to the cloud and the essential clients. A clinical helpful pro needs to pass on and dispatch the EHRs of patients to the sanitizer for managing them in the HER information contraption. Regardless, the ones EHRs as a general rule join the faulty records of impacted individual and prospering office, which consolidate influenced man or woman's name, patient's ID colossal course of action and restorative establishment's name. To keep the privateness of impacted character from the sanitizer, the clinical therapeutic expert will daze the influenced person's sensitive records of every HER sooner than sending this EHR to the sanitizer. The obliging master by then makes marks for this blinded EHR and sends them to the sanitizer. The sanitizer shops those messages into HER bits of learning gadget. Unequivocally when the recuperating therapeutic master needs the EHR, he sends an arrangements to the sanitizer after which the sanitizer downloads the blinded EHR from the EHR estimations device and sends it to the clinical pleasing supportive specialist. Finally, the sharp expert recovers the undeniable EHR from this blinded EHR. Decisively when this EHR ought to be moved and shared inside the cloud for research reason, so you can join the strategy, the sanitizer needs to clean the estimations squares basically like the influenced character's risky data of the EHR. In like way, to watch the security of sanatorium, the sanitizer needs to clean the estimations squares basically like the flourishing office's capricious bits of learning.

\section{Drawbacks}

- According to this concept, out sourcing affected person document does not have any previous permission from affected person.

- There isn't always any choice to outsource simplest authorised patient statistics.

\section{PROPOSED SYSTEM}

Distributed database plays a essential function in day after day lifestyles because within the gift generation, enterprise surroundings is growing at very speedy charge so our simple choice is to get reliable facts from any supply. Since our database is despatched, manner facts is located at unique geographical places and ultimately enables to without problems get right of entry to our valuable \& precious facts. We advise an structure That integrates cloud database offerings with facts integrity and the possibility of executing concurrent operations on encrypted information. It is the solution helping geographically distributed customers to attach at once to an encrypted cloud database, and to execute the concurrent and the unbiased operations which consist of those improving the database form. Distributed database is the rising approach which makes a speciality of concurrency control and protection problems beneath this disbursed database. In this research paintings, information protection is superior via the usage of NTRU (N-th diploma Truncated polynomial Ring Unit or Number Theory Research Unit) uneven key set of guidelines in which the wonderful keys are used for encryption of plaintext and decryption of ciphertext. These keys are named as public and private keys. NTRU being rapid and comfortable hashing set of rules that allows you to offer greater safety to the tool, in terms of throughput and their processing pace. Its number one Characteristics are the low reminiscence and computational requirements as supplying a high safety degree. It is a totally well-organized public-key cryptosystem. 


\section{HYBRID CLOUD STORAGE FOR SECURE AUTHORIZATION AND INFORMATION HIDING}

NTRU (N-th affirmation Truncated polynomial Ring Unit) is an open supply and authorized open key cryptosystem which uses cross segment based completely genuinely cryptography for encryption and unscrambling of reports. The keys used on this plan of rules are: open keyand private key. The key's used for the encryption is Public Key or to assert the propelled imprint yet private secret's used for interpreting or to make automated mark, as presented in Figure 3.. [10]

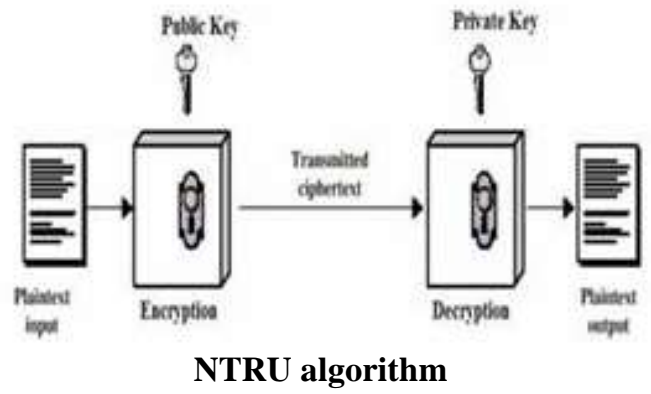

It is essentially established on polynomial science; in this manner it gives particularly brisk count for the encryption and unscrambling of the message. NTRU has a ton less unpredictability. The undertakings depend generally on gadgets which are in a polynomial ring:

The polynomials, gift in the ring have integer coefficients and degree $\mathrm{N}-1$ :

$$
\mathrm{a}=\mathrm{a}_{\mathrm{O}}+\mathrm{a}_{1} \mathrm{X}+\mathrm{a}_{2} \mathrm{X}^{2}+\ldots \ldots+\mathrm{a}_{\mathrm{N}-2} \mathrm{X}^{\mathrm{N}-2}+\mathrm{a}_{\mathrm{N}-1} \mathrm{X}^{\mathrm{N}-1}
$$

Addresses the most extraordinary degree N-1 for all of the polynomials in the ring $\mathrm{R}$, little and colossal modulus independently, $\mathrm{N}$ is thought as uneven, in which $\mathrm{p}$ and $\mathrm{q}$ are co-top. Expect $\mathrm{f}, \mathrm{g}, \mathrm{r}, \mathrm{e}$, and an are all in all ring polynomials.

A. Key Generation: NTRU fuses an open key and an individual key. The open riddle is used for scrambling message and can be appeared to all people. Messages mixed with this key can best be decoded in a decrepit proportion of time using the individual key.

B. Encryption: For encryption of a plaintext message $m \varepsilon$ $\mathrm{R}$ the use of $\mathrm{h}$ as the lion's offer key, Alice picks a self-assertive detail $r \varepsilon R$ and makes the figure printed content: $\mathrm{e} \equiv \mathrm{r} * \mathrm{~h}+\mathrm{m}(\bmod \mathrm{q})$

C. Decryption: For deciphering of the figure content $\mathrm{e}$ using the $\mathrm{f}$ as a private key, Bob in any case forms the charge:

$$
\mathrm{a} \equiv \mathrm{f} * \mathrm{e}(\bmod \mathrm{q})
$$

Impact by then picks a $\varepsilon \mathrm{R}$ to satisfy this closeness and to lie in a super fine pre-accurate subset of $\mathrm{R}$. He resulting does the $\bmod \mathrm{p}$ estimation $\mathrm{fq}-1 * \mathrm{a}(\bmod \mathrm{p})$ and the rate he figures is relative to $\mathrm{m}$ modulo $\mathrm{p}$. The fundamental tendencies of NTRU set of rules are low computational and memory necessities for introducing an unbelievable stage security. In this arrangement of standards the issue is scanned for the length of the factorization of the polynomials into two considered one in everything about sort polynomials having especially less coefficients. NTRU is a plainly usable, pleasingly practiced and promising cryptosystem..

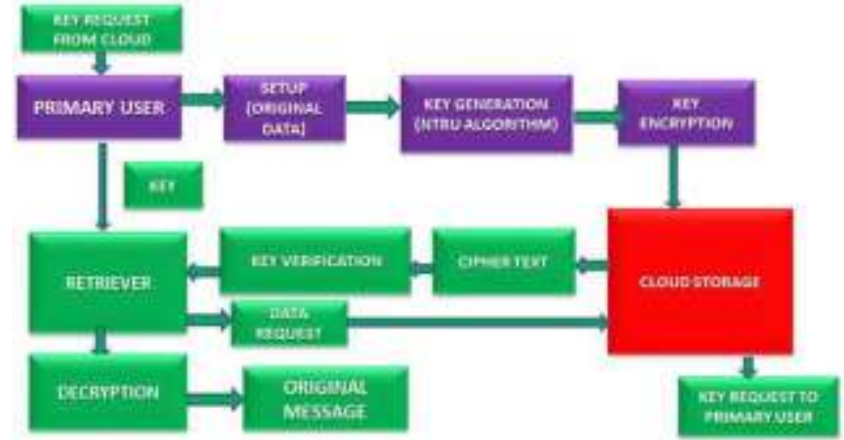

Fig 3 Proposed system block diagram

List of Modules

Aggregate Key

This changed into completed thru the facts owner to randomly generate a public/grasp-secret key pair. This key completed thru the unmarried individual. In case the retriever itself shares the vital component to others it does now not paintings. This key generated for anyone by the statistics proprietor as properly as it does not maintained through the cloud service issuer. This key must be managed via individual. The cloud provider company shops the crucial factor for authentication motive best.

User

User is the records proprietor he/she generates the data and uploads to cloud and generates key for retriever. All the statistics's need to be encrypted at the same time as record importing technique. This encryption technique is finished by using the use of absolutely everyone who desires to encrypt statistics. On input a public-key pk, an index $\mathrm{i}$ denoting the cipher textual content beauty, and a message $\mathrm{m}$, it outputs a cipher textual content $\mathrm{C}$. But in our mission this technique for the consumer.

\section{Setup Phase}

Executed thru the statistics Proprietor to setup an account on an un viable server. On input a protection level parameter and the variety of cipher textual content lessons n (i.E., class index need to be an integer bounded with the aid of 1 and $n$ ) it outputs the general public system parameter parameters, this is unnoticed from the input of the alternative algorithms for brevity.

\section{Retriever}

The retriever is the person who wants to view the uploaded data. The data is associated with attributes so the authenticated retrievers only view the original data. To authenticate the retriever we have verification phase in that retriever credentials are verified. After that while the retriever searching and view the data they should have corresponding aggregate key. 


\section{RESULT VIEW}

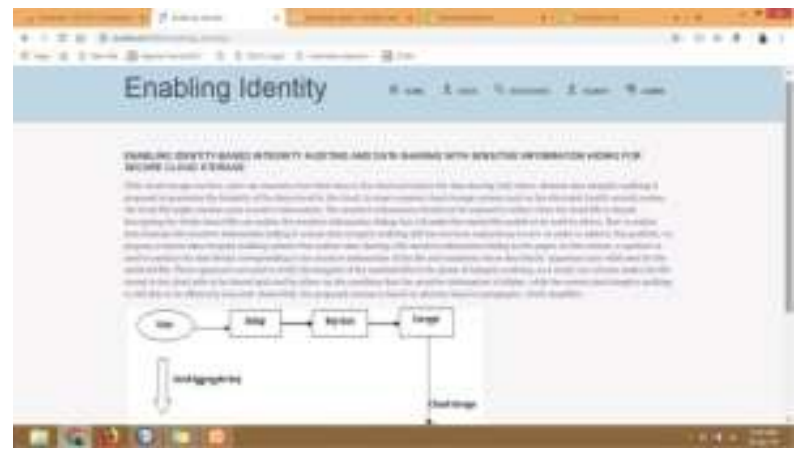

User Login
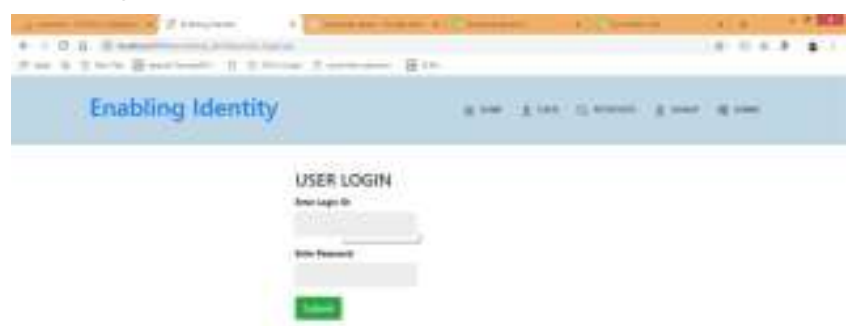

\section{Retriever Login}

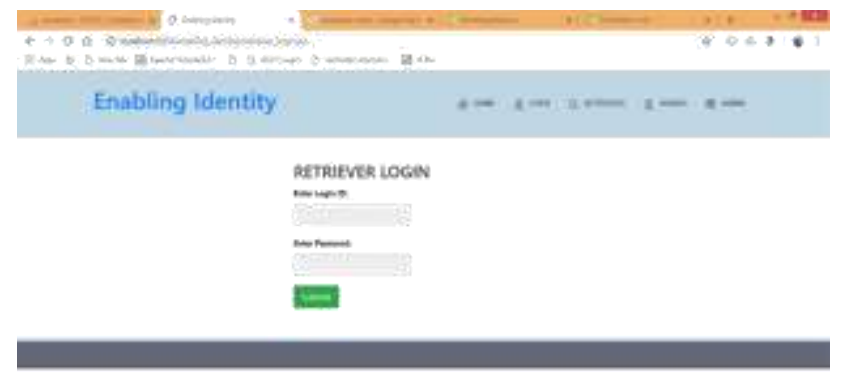

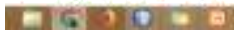

User setup

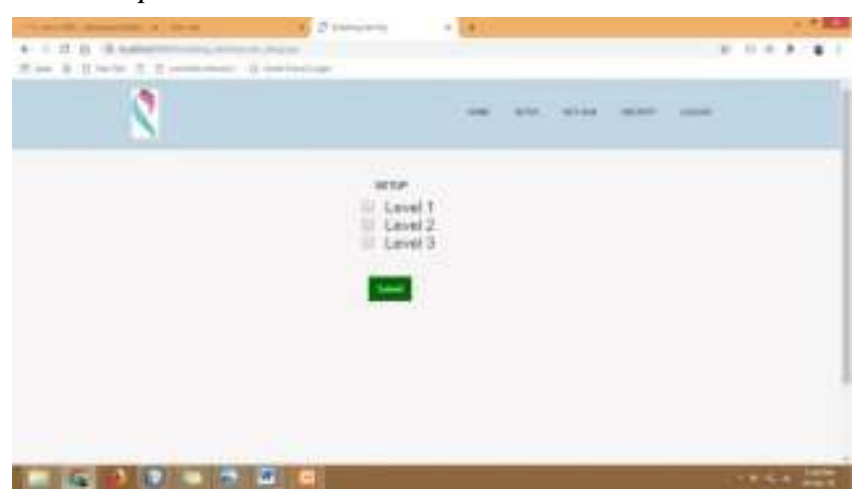

User key Generation

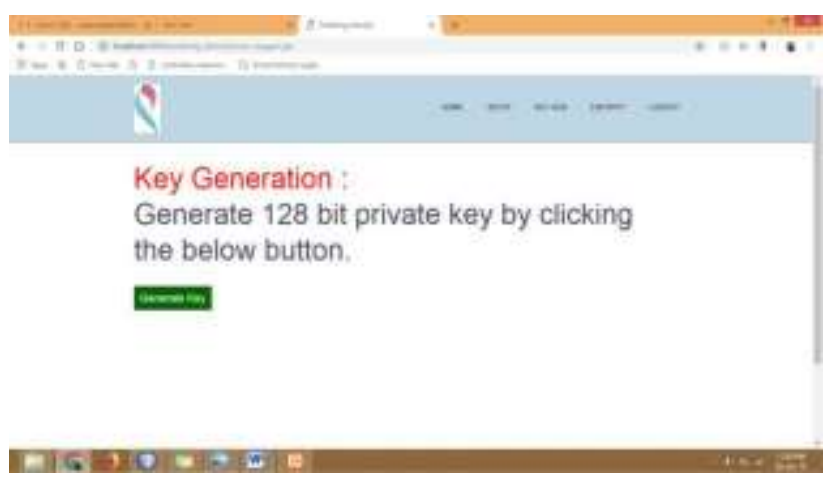

User share and encrypt file

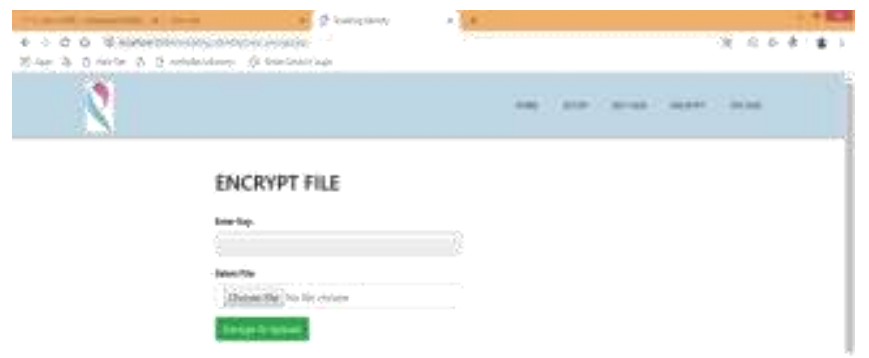

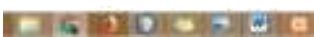

Retriever view file

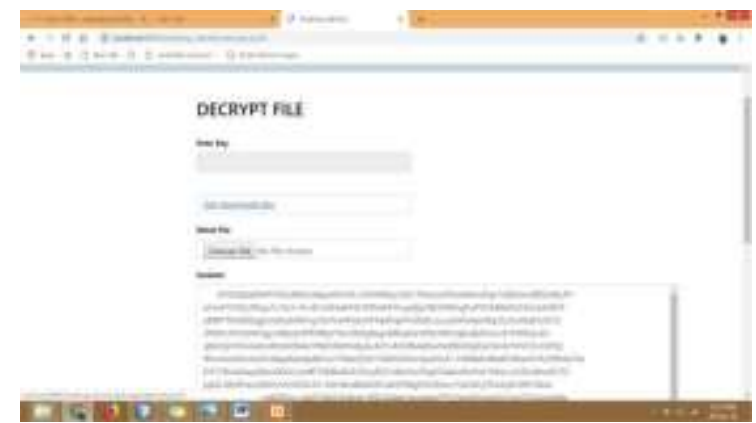

Retriever get aggregate key

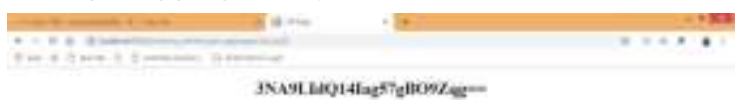


issues. Security performs crucial function on this work as to defend our touchy statistics from the unauthorized user. This dissertation has implemented the NTRU algorithm in net beans. In this research we have studied the existing Ramp Secret Sharing Scheme used for encryption and decryption of data and to improve the reliability of distributed deduplication system. But there are some limitations of existing RSSS technique to overcome the limitations of existing technique NTRU algorithm is used.

The proposed technique requires less time for encryption and decryption of data while sharing the files in distributed database system and also takes less time for authentication purpose.

\section{REFERENCES}

1. Q. Zhang, L. Cheng, and R. Boutaba, "Cloud computing: state-of-the-art and research challenges," J. Internet Ser. Appl., vol. 1, 2010, pp. 7-18.

2. M. I. Williams, A Quick Start Guide to Cloud Computing: Moving Your Business into the Cloud. Kogan Page, 2010.

3. M.D. Ryan, "Cloud computing security: The scientific challenge, and a survey of solutions," J. Syst. Softw., vol. 86 (9), Sept. 2013, pp. 2263-2268.

4. M. Factor et al., "Secure Logical Isolation for Multi-tenancy in cloud storage," IEEE 29th Sym. on Mass Storage Systems and Technologies (MSST), 2013, pp. 1-

5. F. Sabahi, "Virtualization-level security in cloud computing," IEEE 3rd Intl. Conf. on Communication Software and Networks, pp. 250-254, 2013.

6. X. Chen, J. Andersen, Z.M. Mao, M. Bailey, and J. Nazario, "Towards an understanding of anti-virtualization and antidebugging behavior in modern malware," IEEE Intl. Conf. on Dependable Systems and Networks With FTCS and DCC (DSN), 2008, pp. 177-186.

7. S. Vijayakumar, Q. Zhu, and G. Agrawal, "Dynamic Resource Provisioning for Data Streaming Applications in a Cloud Environment," IEEE Second Intl. Conf. on Cloud Computing Technology and Science, 2010, pp. 441-448.

8. F. Rocha, S. Abreu, and M. Correia, "The Final Frontier: Confidentiality and Privacy in the Cloud,"

9. IEEE Computer, vol. 44 (9), Sept. 2011, pp. 44-50.

10. S. Pearson, "Taking account of privacy when designing cloud computing services," ICSE W. on Software Engineering Challenges of Cloud Computing, 2009, pp. 44-52.

11. "The CIA Principle" available at http://www.doc.ic.ac.uk/ ajs300/security/CIA.html [Accessed: 15 Mar. 2017]

12. S. Singh, Y.-S. Jeong, and J. H. Park, "A survey on cloud computing security: Issues, threats and solutions," J. Netw. Comput. Appl., vol. 75, Nov. 2016, pp. 200-222.

13. N. Gruschka and M. Jensen, "Attack Surfaces: A Taxonomy for Attacks on Cloud Services," IEEE 3rd Intl. Conf. on Cloud Computing, 2010, pp. 276-279. 